\title{
An Analysis of Blood Grouping Discrepancies : Study From Tertiary Hospital based Blood Bank in Vadodara
}

\author{
Ashu Dogra ${ }^{1}$, Devanshi Gosai ${ }^{2}$ \\ ${ }^{1}$ Associate Professor, Deptt of Pathology, SBKSMIRC, Sumandeep University, ${ }^{2}$ Assistant Professor, Department of \\ Transfusion Medicine SBKSMIRC, Sumandeep University
}

\begin{abstract}
Background:-ABO and Rhesus blood group system is considered as clinically significant blood group systems in transfusion practice. A feature of the $\mathrm{ABO}$ system is the regular occurrence of anti $\mathrm{A}$ and anti $\mathrm{B}$ in the absence of the corresponding red cell antigen. A Discrepancy exists when results of forward testing does not match with reverse testing. Blood donors and patients must be correctly $\mathrm{ABO}$ and $\mathrm{Rh}$ grouped because transfusing $\mathrm{ABO}$ incompatible blood transfusions which may lead even death of patient.
\end{abstract}

Aims and Objectives To assess the incidence and cause of blood grouping discrepancies in Blood Bank at Tertiary care teaching hospital during Jan 2019 to December 2019.

Methods : Forward and reverse grouping were performed on blood samples from inpatients ,outpatients and donors during the study period. ABO discrepancies were studied with their clinical details to group their discrepancies and resolve them with suitable steps.

Results: A total of 10,048 (patients and donors) who satisfied inclusion criteria were included and $\mathrm{ABO}$ typed among which there were 55 discrepancies were observed (15 donors and 35 patients). The problem in patients was due to expression of weak antigens. The problem in patients were due to clinical conditions, the most common being autoimmune haemolytic anaemia.

Conclusions: The ABO blood group discrepancy reported in present study is $0.49 \%$. The study helped to determine the incidence and causes of discrepancy encountered in tertiary care hospital. Discrepant results should be recorded, and resolved by suitable measures and correct ABO \& Rh Blood group should be released to avoid any mismatched transfusions.

Key words: $A B O$ discrepancy, Blood transfusion, Rhesus group.

\section{Introduction}

$\mathrm{ABO}$ and Rhesus blood group system, also known as Major Blood group system is reported to be one of the most important clinically significant Blood Group system. Blood donors and patients needs to be correctly $\mathrm{ABO}$ and $\mathrm{Rh}$ grouped because transfusing $\mathrm{ABO}$ -incompatible blood may result in transfusion reaction which may lead even to death of patient. ${ }^{(1)}$

For ABO Blood group of an individual it is important to do both cell grouping ( forward typing ) and serum grouping ( reverse typing). In forward grouping, the unknown test cells are antigen typed against known group A and group B cells.

Both forward and reverse typing results must match to confirm the true ABO type of an individual. ${ }^{(2)}$.

ABO typing needs to be performed correctly for the safety of patient and to avoid adverse transfusion reactions. The risk reported due to acute haemolytic transfusion reaction because of incompatible blood components is about 100 times more than the risk reported due to transfusion transmitted infections. (3) 
In $\mathrm{ABO}$ discrepancies forward blood group does not match with the reverse blood group. In such cases interpretation of final $\mathrm{ABO}$ Blood group type must be delayed till the discrepancy is resolved. In clinical emergencies, generally group $\mathrm{O}$ negative Red Blood Cells are transfused to save the life of patient ${ }^{(4)}$

Table $1^{(5)}$ : ABO Discrepancies are Classified Into Four Types.

\begin{tabular}{|c|c|}
\hline Group 1 & Missing or weak antibodies. \\
\hline Group 2 & Missing or weak antigens. \\
\hline Group 3 & Rouleaux formation and pseudo agglutination \\
\hline Group 4 & Miscellaneous. \\
\hline
\end{tabular}

\section{Aims and Objectives}

To analyse the blood grouping discrepancies reported in Teaching hospital based blood bank during Jan2019 to December 2019.

\section{Material \& Methods}

A retrospective study was carried out in Dhiraj Blood Bank attached with SBKSMIRC, Vadodara, from January 2019 to December 2020. An analysis of ABO discrepancy was done on patients and Donors samples. Forward and reverse grouping were performed on blood samples from inpatient, outpatient and donors.

Inclusion criteria: All patients and donors samples with EDTA or citrated anticoagulated blood for forward grouping and clotted blood sample for reverse grouping.

Exclusion criteria: Hemolysed samples and clotted samples of newborn upto 3 months of age for reverse grouping.

The protocol used by blood bank includes following determinations:

$\mathrm{ABO} \mathrm{Rh}(\mathrm{D})$ group , $\mathrm{ABO}$ group is determined by $\mathrm{ABO}$ gel grouping card which contains antisera $\mathrm{A}$ antisera B impregnated in gel columns for forward grouping and neutral gel cards for testing expected antibodies with A and B red cells in reverse grouping .(Tulip Anti A Anti B Anti D Control Reverse diluents gel card for gel card grouping were used)

Antibody screen testing for unexpected antibodies for RBCs transfusion with antiglobulin test incubated at $37 * \mathrm{C}$

The blood bank standard operating procedures and Quality manual mandates that all results are compared with patients records filed in blood bank.

ABO discrepancies were detected on comparing the patients forward blood group results with reverse blood group results.

For A subgroup anti A lectin is used, Dolichos biflorus in the diluted state, which reacts with A1 and $\mathrm{A} 1 \mathrm{~B}$ red cells but not with $\mathrm{A} 2$ and $\mathrm{A} 2 \mathrm{~B}$ cells. If red cell agglutinate the person is subgroup A1 .If no agglutination takes place, person is A2.

IgM alloantibodies such as Anti- Le ${ }^{\mathrm{a}}$, Anti P1, Anti-M, and Anti $\mathrm{N}$ may cause a serum mediated discrepancy with reverse ABO grouping cells. Antibody screen and identification are needed in such cases, followed by selection of blood that lacks antigen.

Discrepancies were solved according to discrepancy type protocol. 


\section{Results and Discussion}

In present study on ABO discrepancy 10,048 (Patient and Donor ) who satisfied inclusion criteria were included and ABO typed. There were $0.49 \%$ discrepancies observed. 15 were of donors and 35 were of patients.

In donors incidence of discrepancies were found to be 15 out of 10,048 . Out of 15 discrepancies there were only males in age group of 21-30 years.

Among 15 cases on grouping them on the type of discrepancies 10 out of 15 cases were found to be major category. Group 2 comprises $13.3 \%$ of total ( 2 out of 15 ) and group 4 only $20 \%$.( 3 out of 15 ).

No discrepancy came to group 3 category in donors.

Table 2 : Causes of discrepancy in donors

\begin{tabular}{|c|c|c|c|}
\hline S.No. & Groups & Causes & Number \\
\hline 1 & Group 1 & $\begin{array}{l}\text { Weak expression of } \mathrm{Rh} \text { antigen } \\
\text { Weak expression of } \mathrm{B} \text { antigen }\end{array}$ & $\begin{array}{l}5 \\
1\end{array}$ \\
\hline 2 & Group 2 & $\begin{array}{c}\text { Bombay blood group } \\
\text { A2B } \\
\text { Weak expression of antibody }\end{array}$ & $\begin{array}{l}1 \\
2 \\
2\end{array}$ \\
\hline 3 & Group 3 & - & \\
\hline 4 & Group 4 & ICT Positive. & 4 \\
\hline
\end{tabular}

In Patients :-Forward and reverse typing were done on both inpatient and outpatient and those which showed discrepancies were resolved by suitable measures.

Out of 6664 Patients 35 (0.52\%) patients showed discrepancy.
Among patients discrepancies were found to be more in females $(71.4 \%)$ than in males $(28.57 \%)$. Among the 35 patients age group distribution of discrepancy were calculated and found to be more in age group of 0-9 years i.e 20 out of 35 . This is mainly due to absence of / reduced development of antibodies in infants $>3$ months old. 
Table 3 : Types of discrepancies in patients

\begin{tabular}{|c|c|c|}
\hline Cases & Causes & No. of patients \\
\hline \multirow{3}{*}{ Group 1 } & Neonates & 10 \\
& Weak expression of antigen & 10 \\
\hline \multirow{3}{*}{ Group 2 } & Subgroups & 2 \\
& Weak expression of antibody & 1 \\
& Infection & 1 \\
& Malignancy & 2 \\
\hline Group 3 & Multiple myeloma & 1 \\
& Abnormal plasma proteins & 2 \\
\hline Group 4 & & 1 \\
& AIHA & 2 \\
\hline
\end{tabular}

The present study was designed to determine the incidence and causes of all ABO discrepancies detected in tertiary care hospital blood bank. Blood Donors and patients must be correctly $\mathrm{ABO}$ and $\mathrm{Rh}$ grouped because transfusing $\mathrm{ABO}$ incompatible blood may result in transfusion reaction which may lead to death of patient ${ }^{(1)}$

\section{Discrepancies in Donors}

Findings of this one year study showed that in donor population the causes of discrepancies were very much different from that of patient population. In donors major cause were found to be weak expression of antigen/ antibody whereas in patients the problem involved atypical antibodies and acquired antigens creating blood group changes.

In donors it was found that most common discrepancy was weak expression of antigen (mainly Rh) and weak antibody. In study by Srikrishna et.al found that about 1 in 1000 persons face the problem of weak D phenotype which are reported as Rh negative in some blood banks/ Laboratories . hence uniform and consistent standards for reporting are required ${ }^{(6)}$

Other observation was discrepancies in donor population mostly seen in age group of 21-30 years and in patient population was found in age group of0-9 years. Reason may be due to age limit in donors which contributes to maximum donors in this age group and thus increase in incidence of discrepancies.

In patient population females are found to have more incidence of discrepancies (57.1\%) than males. Reason is exposure to antigens in pregnancy, pregnancy related transfusions, predominant occurrence of autoimmune disease in female patients. There are 20 cases in the age group.

The acquired B phenotype arises in vivo in patients with bacterial infections when bacteria produces deacetylase enzymes which chemically alters terminal sugar of A antigen ( $N$ acetyl D Galactosamine) so that it resembles B determining galactose. In present study one case of infection was reported which included Gram negative sepsis . 
There was one case of multiple myeloma who showed elevated levels of globin chains which result in rouleaux formation.

Leukaemia is another important condition for ABO discrepancies . Reason for discrepancy is hypogammaglobulinemia.

Similar case has been reported by Lenz and curie with a patient who had long history of pneumonia who showed discrepancy in forward and reverse typing by not showing reaction with $\mathrm{B}$ cells even after extended incubation . the cause of this is hypogammaglobulinemia which result in immunodeficiency.(7)

Subgroups comprise of major part of group 3 discrepancy 2 out of 35-. Among subgroups there were more incidence of $\mathrm{A} 2$ and $\mathrm{A} 2 \mathrm{~B}$ groups --two- cases and Bombay group-one case-. These were corrected by suitable lectins.

AIHA and multiple transfusions are leading cause of ABO Discrepancy, autoantibody in AIHA can be warm/ cold which are coated on red cells that produce positive coombs test result. This may promote weak agglutination in forward grouping, resulting in ABO discrepancy . Although AIHA may present with an ABO-Rh typing discrepancy, accurate blood group identification usually can be determined without specialized techniques.

\section{Conclusion}

The incidence of $\mathrm{ABO}$ Discrepancy in present study reported was $0.49 \%$. The incidence reported in donor population was 15 of 50 and in patents incidence of $\mathrm{ABO}$ discrepancy reported was 35 out of 50 . For safe blood transfusion to patients, it is imperative that all blood group discrepancies must be resolved correctly

Ethical Clearance: Taken from ethical committee SBKSMIRC

\section{Source of Funding: Self}

Conflict of Interest : Nil.

\section{References}

1. Makroo R.N. ABO Blood group system: Compendium of transfusion medicine. $\mathrm{I}^{\text {st }}$ Edition. NewDelhi: Alps Printers; 1999:28-32.

2. Shahshahani HJ, Hayati A. Blood group Discrepancies at a Regional Blood Centre. International Journal of Hematology -Oncology and stem cell Research.2020;14(1):39-44.

3. Chiaroni J, Legrand D, Dettori I, et al. Analysis of ABO Discrepancies occurring in 35 French Hospitals. Transfusion .2004; 44(6) :860-4

4. Cooling L. ABO,H, Lewis. Blood Groups and structurally related antigens. In: Fung MK, Grossman BJ, Hillyer CD , et al., editors. Technical Manual. $18^{\text {th }}$ Edition. Bethesda, Maryland : American Association of Blood Bank; 2014.297313

5. Nepal B, Shrestha B, Maharjan S, et al. ABO Blood group discrepancies : study of prevalence and related factors. Grand Medical Journal: 2019;1(2): 88-91.

6. Deepthi Krishna G, Sreedhar Babu KV, et al. A study on Rh incompatibility and frequency of weak D among blood donors and patients at a tertiary care referral teaching hospital in Tirupati. Journal of Clinical Science Research. 2015;4:281-4.

7. Walter $\mathrm{J}$, Linz and Robert $\mathrm{T}$ Curie. $\mathrm{ABO}$ Discrepancy: Reverse type, Transfusion Journa.2007;47:1-3.

8. E. Anne Stiene- Martin et al . Acquired Immune Anemias of increased destruction: Clinical Hematology, Principles, Procedures. ${ }^{\text {nd }}$ Edition. Lippincott publishers; NewYork : 285. 